\title{
Pacific
}

Journal of

Mathematics

\section{CONVOLUTION AND LIMIT THEOREMS FOR CONDITIONALLY FREE RANDOM VARIABLES}

Marek Bożejko, Michael Leinert AND Roland SPeicher 


\title{
CONVOLUTION AND LIMIT THEOREMS FOR CONDITIONALLY FREE RANDOM VARIABLES
}

\author{
Marek Bożejko, Michael Leinert and Roland Speicher
}

\author{
Dedicated to Professor Wilhelm von Waldenfels \\ on the occasion of his 65th birthday
}

\begin{abstract}
We introduce the notion of a conditionally free product and conditionally free convolution. We describe this convolution both from a combinatorial point of view, by showing its connection with the lattice of non-crossing partitions, and from an analytic point of view, by presenting the basic formula for its $R$-transform. We calculate explicitly the distributions of the conditionally free Gaussian and conditionally free Poisson distribution.
\end{abstract}

\section{Introduction.}

In [BSp], we introduced a generalization with respect to two states of the reduced free product of Voiculescu [Voi1, VDN] and gave some preliminary results on this concept. Here, we want to examine this notion more systematically, in particular, we want to investigate the corresponding convolution. We describe this convolution both from a combinatorial point of view - by showing its connection with the lattice of non-crossing partitions - and from an analytic point of view - by presenting the basic formula for its $R$-transform, which is the replacement of the classical Fourier-transform. We calculate explicitly the distributions of the corresponding Gaussian and Poisson law by a careful examination of the structure of the non-crossing partitions.

Instead of the terms " $\psi$-independence" and " $\psi$-product" of [BSp], we will use here the more precise expressions "conditionally free" and "conditionally free product", or just the abbreviation "c-free".

Let us start with a motivation for our concept of "c-freeness". Consider a group $G=*_{i \in I} G_{i}$ which is the free product of groups $G_{i}(i \in I)$, i.e. each element $g \neq e$ of $G$ can be written uniquely in the form $g=g_{1} \ldots g_{n}$, where $e \neq g_{j} \in G_{i(j)}$ and $i(1) \neq i(2) \neq \cdots \neq i(n)$. To see the nature of this 
decomposition of $G$ more clearly, we state it in a more abstract way by using the functions $\psi_{i}=\delta_{e}$ on $G_{i}$, i.e. $\psi_{i}: G_{i} \rightarrow \mathbb{C}$ with $\left(g \in G_{i}\right)$

$$
\psi_{i}(g)= \begin{cases}1, & g=e \\ 0, & g \neq e .\end{cases}
$$

Then the above decomposition has the form: Each element $g \neq e$ can be written as $g=g_{1} \ldots g_{n}$, where $g_{j} \in G_{i(j)}, i(1) \neq i(2) \neq \cdots \neq i(n)$ and $\psi_{i(j)}\left(g_{j}\right)=0$ for all $j=1, \ldots, n$.

If we are now given functions $\varphi_{i}: G_{i} \rightarrow \mathbb{C}$ with $\varphi_{i}(e)=1$, then we can form their c-free product in the canonical way, namely we define a new function $\varphi=*_{i \in I}\left(\varphi_{i}, \psi_{i}\right)$ on $G$ by $\varphi(e)=1$ and

$$
\varphi(g):=\varphi_{i(1)}\left(g_{1}\right) \ldots \varphi_{i(n)}\left(g_{n}\right),
$$

if $g \neq e$ has the above representation. The key property of this construction is the fact that, if the $\varphi_{i}$ are positive definite on $G_{i}$, then $\varphi$ is positive definite on $G=*_{i \in I} G_{i}$, see [Boz1, Boz2].

As an example of such a c-free product one can take each $G_{i}$ as a copy of $\mathbb{Z}$ and $\varphi_{i}$ as $\varphi_{i}(k)=\exp (-\lambda|k|)(k \in \mathbb{Z})$ for some $\lambda>0$. Then $G$ is the free group on $|I|$ generators and $\varphi$ is given by $\varphi(g):=\exp (-\lambda|g|)$, where $g \mapsto|g|$ is the canonical length function on the free group. Since the $\varphi_{i}$ are positive definite functions on $\mathbb{Z}$, this $\varphi$ is also positive definite. This property of the length function on the free group was proven by Haagerup [Haa].

If we translate the above description of $\varphi$ from groups to group algebras, then it reads in the following way: Let $\mathcal{A}_{i}:=\mathbb{C} G_{i}$ and $\mathcal{A}:=\mathbb{C} G$ be the group algebras of $G_{i}$ and $G$, respectively. Then, given linear functionals $\varphi_{i}$ on $\mathcal{A}_{i}$ with $\varphi_{i}(1)=1$, we can define a linear functional $\varphi=*_{i \in I}\left(\varphi_{i}, \psi_{i}\right)$ on $\mathcal{A}$ by $\varphi(1)=1$ and the characterizing property

$$
\varphi\left(a_{1} \ldots a_{n}\right)=\varphi_{i(1)}\left(a_{1}\right) \ldots \varphi_{i(n)}\left(a_{n}\right),
$$

whenever $a_{j} \in \mathcal{A}_{i(j)}, i(1) \neq i(2) \neq \cdots \neq i(n)$ and $\psi_{i(j)}\left(a_{j}\right)=0$, where $\psi_{i}$ is now the linear extension of $\delta_{e}$ to $\mathcal{A}_{i}$.

In this formulation it is unnatural to restrict to $\mathcal{A}_{i}=\mathbb{C} G_{i}$ and to $\psi_{i}=\delta_{e}$, one can now consider the above c-free product for arbitrary unital algebras $\mathcal{A}_{i}$ and arbitrary states $\psi_{i}$ on $\mathcal{A}_{i}$. One of the main results in [BSp] was that also in this general case $\varphi$ is a state if the $\varphi_{i}$ are. This was proved by $\bar{a}$ explicit construction of the corresponding c-free product. We will give in Sect. 2 another, purely algebraic, proof of this basic fact.

After this basic considerations we will then switch to the corresponding notion of c-free convolution, the main topic of our investigations. Since 
compactly supported measures $\mu$ on $\mathbb{R}$ are determined by their moments, such measures can be identified with states on the polynomial algebra $\mathbb{C}\langle X\rangle$. Thus we can characterize our convolution in the following way. Given pairs of compactly supported probability measures $\left(\mu_{1}, \nu_{1}\right)$ and $\left(\mu_{2}, \nu_{2}\right)$, we define their c-free convolution by the following prescription: Consider $\mathcal{A}_{1}=\mathbb{C}\left\langle X_{1}\right\rangle$ and $\mathcal{A}_{2}=\mathbb{C}\left\langle X_{2}\right\rangle$. Then $\mathcal{A}=\mathcal{A}_{1} * \mathcal{A}_{2}=\mathbb{C}\left\langle X_{1}, X_{2}\right\rangle$. We have on $\mathcal{A}_{i}$ the states $\mu_{i}$ and $\nu_{i}$, thus our construction of a c-free product gives a state $\varphi=$ $\left(\mu_{1}, \nu_{1}\right) *\left(\mu_{2}, \nu_{2}\right)$ on $\mathcal{A}$. If we restrict this state to $\mathbb{C}\langle X\rangle$, where $X=X_{1}+X_{2}$, then the distribution of $X$ determines a measure $\mu$, which we call the c-free convolution of $\left(\mu_{1}, \nu_{1}\right)$ and $\left(\mu_{2}, \nu_{2}\right)$, denoted by $\mu=\left(\mu_{1}, \nu_{1}\right) \boxplus\left(\mu_{2}, \nu_{2}\right)$. The name "c-free convolution" indicates that $\mu$ is the distribution of the sum of $X_{1}$ and $X_{2}$, which are distributed according to $\mu_{1}$ and $\mu_{2}$ and which are c-free. If $\nu_{i}=\mu_{i}(i=1,2)$, then our construction reduces to the free convolution of Voiculescu [Voi2].

To be able to talk about associativity, we should also define a new measure $\nu$ and it turns out that the natural candidate for this is the free convolution $\nu_{1} \boxplus \nu_{2}$ of $\nu_{1}$ and $\nu_{2}$, thus

$$
(\mu, \nu)=\left(\mu_{1}, \nu_{1}\right) \boxplus\left(\mu_{2}, \nu_{2}\right),
$$

where

$$
\mu=\left(\mu_{1}, \nu_{1}\right) \boxplus\left(\mu_{2}, \nu_{2}\right), \quad \nu=\nu_{1} \boxplus \nu_{2} .
$$

In Sect. 3, we will examine this c-free convolution from a combinatorial point of view and show that it is, similarly as in the case of the free convolution [Spe2], determined by the lattice of non-crossing partitions.

In Sect. 4, we treat the c-free central and Poisson limit theorem by a careful analysis of the structure of the non-crossing partitions. We will thereby derive some combinatorial identities for these partitions which also have some interest of their own.

In Sect. 5 , we present a systematic machinery for an analytic description of c-free convolution, namely the generalization of Voiculescu's $R$-transform [Voi2].

\section{Definition and positivity of the c-free product.}

We shall work in the category of unital $*$-algebras and states. By a state $\varphi$ on a unital $*$-algebra $\mathcal{A}$ we will always mean a linear functional $\varphi: \mathcal{A} \rightarrow \mathbb{C}$, which is normalized $(\varphi(1)=1)$, hermitian $\left(\varphi\left(a^{*}\right)=\overline{\varphi(a)}\right.$ for all $\left.a \in \mathcal{A}\right)$ and positive $\left(\varphi\left(a a^{*}\right) \geq 0\right.$ for all $\left.a \in \mathcal{A}\right)$. 
Let now $\mathcal{A}_{i}(i \in I)$ be unital $*$-algebras equipped with a pair of states $\left(\varphi_{i}, \psi_{i}\right)$. Then we want to define a new state $\varphi=*_{i \in I}\left(\varphi_{i}, \psi_{i}\right)$ on the algebraic free product $\mathcal{A}=*_{i \in I} \mathcal{A}_{i}$ (identification of the units is assumed). Observing that with the decompositions $\mathcal{A}_{i}=\mathbb{C} 1 \oplus \mathcal{A}_{i}^{o}$, where $\mathcal{A}_{i}^{o}:=\operatorname{ker} \psi_{i}$, one can identify $\mathcal{A}$ as a vector space with

$$
\mathbb{C} 1 \oplus \bigoplus_{n=1}^{\infty} \bigoplus_{i(1) \neq \cdots \neq i(n)} \mathcal{A}_{i(1)}^{o} \otimes \cdots \otimes \mathcal{A}_{i(n)}^{o}
$$

it is clear that we can define uniquely and consistently a linear functional $\varphi=*_{i \in I}\left(\varphi_{i}, \psi_{i}\right)$ on $\mathcal{A}$ by $\varphi(1)=1$ and the following characterization:

$$
\varphi\left(a_{1} \ldots a_{n}\right)=\varphi_{i(1)}\left(a_{1}\right) \ldots \varphi_{i(n)}\left(a_{n}\right),
$$

whenever

$$
a_{j} \in \mathcal{A}_{i(j)}, \quad i(1) \neq i(2) \neq \cdots \neq i(n), \quad \psi_{i(j)}\left(a_{j}\right)=0 .
$$

Such elements $a_{1} \ldots a_{n} \in \mathcal{A}_{i(1)}^{o} \otimes \cdots \otimes \mathcal{A}_{i(n)}^{o}$ will be called elementary elements in the following.

Of course, the main problem is now to see that $\varphi$ is positive. In [BSp], this was proven by an explicit construction of the GNS representation of $\mathcal{A}$ with respect to $\varphi$. Here, we want to give a purely algebraic proof of this fact. For this we need a lemma about the calculation of $\varphi$.

Lemma 2.1. Consider two elementary elements

$$
y_{1}=a_{1}^{(1)} \ldots a_{n}^{(1)} \quad \text { and } \quad y_{2}=a_{1}^{(2)} \ldots a_{m}^{(2)} .
$$

(1) If $a_{1}^{(1)}$ and $a_{1}^{(2)}$ do not belong to the same $\mathcal{A}_{i}^{o}$ then

$$
\varphi\left(y_{1}^{*} y_{2}\right)=\varphi\left(y_{1}^{*}\right) \varphi\left(y_{2}\right)
$$

(2) Consider $a \in \mathcal{A}_{i}$ for some $i \in I$. If $a_{1}^{(1)}$ and $a_{1}^{(2)}$ do not belong to $\mathcal{A}_{i}^{o}$ then

$$
\varphi\left(y_{1}^{*} a y_{2}\right)=\psi_{i}(a) \varphi\left(y_{1}^{*} y_{2}\right)-\psi_{i}(a) \varphi\left(y_{1}^{*}\right) \varphi\left(y_{2}\right)+\varphi_{i}(a) \varphi\left(y_{1}^{*}\right) \varphi\left(y_{2}\right)
$$

Proof. (1) Clear, since

$$
\varphi\left(y_{1}^{*} y_{2}\right)=\varphi\left(a_{n}^{(1) *}\right) \ldots \varphi\left(a_{1}^{(1) *}\right) \varphi\left(a_{1}^{(2)}\right) \ldots \varphi\left(a_{m}^{(2)}\right)
$$


(2) This follows from

$$
\begin{aligned}
\varphi\left(y_{1}^{*} a y_{2}\right) & =\varphi\left(y_{1}^{*}\left(a-\psi_{i}(a) 1\right) y_{2}\right)+\psi_{i}(a) \varphi\left(y_{1}^{*} y_{2}\right) \\
& =\varphi\left(y_{1}^{*}\right) \varphi_{i}\left(a-\psi_{i}(a) 1\right) \varphi\left(y_{2}\right)+\psi_{i}(a) \varphi\left(y_{1}^{*} y_{2}\right) \\
& =\varphi\left(y_{1}^{*}\right) \varphi_{i}(a) \varphi\left(y_{2}\right)-\varphi\left(y_{1}^{*}\right) \psi_{i}(a) \varphi\left(y_{2}\right)+\psi_{i}(a) \varphi\left(y_{1}^{*} y_{2}\right)
\end{aligned}
$$

Theorem 2.2. If $\varphi_{i}$ and $\psi_{i}$ are states for all $i \in I$, then $\varphi=*_{i \in I}\left(\varphi_{i}, \psi_{i}\right)$ is a state, too.

Proof. We will show

$$
\varphi\left(x^{*} x\right) \geq|\varphi(x)|^{2} \quad \text { for all } x \in \mathcal{A} .
$$

We can write each $x \in \mathcal{A}$ in the form

$$
x=\alpha 1+\sum_{k} a_{1}^{(k)} \ldots a_{n(k)}^{(k)}
$$

where $\alpha \in \mathbb{C}$ and $a_{1}^{(k)} \ldots a_{n(k)}^{(k)}$ are elementary elements (with $n(k) \geq 1$ ) for all $k$. It suffices to prove the asserted inequality for $x$ without term of the form $\alpha 1$, i.e. we can assume $x$ to be of the form

$$
x=\sum_{k} a^{(k)} y^{(k)}
$$

with

$$
a^{(k)}:=a_{1}^{(k)} \quad \text { and } \quad y^{(k)}:=a_{2}^{(k)} \ldots a_{n(k)}^{(k)} .
$$

Our proof will be by induction on the length of $x$ (i.e. the maximal $n(k)$ in the above representation), and we assume now the validity of the assertion for elements of a smaller length than $x$, in particular for the $y^{(k)}$ and linear combinations of them.

Put now

$$
x_{i}:=\sum_{\substack{k \text { with } \\ a^{(k)} \in \mathcal{A}_{i}}} a^{(k)} y^{(k)} .
$$

Then it suffices to prove the assertion for all $x_{i}$, because this implies, by the first part of Lemma 2.1.

$$
\varphi\left(x^{*} x\right)=\sum_{i, j} \varphi\left(x_{i}^{*} x_{j}\right)
$$




$$
\begin{aligned}
& =\sum_{i=j} \varphi\left(x_{i}^{*} x_{i}\right)+\sum_{i \neq j} \varphi\left(x_{i}^{*} x_{j}\right) \\
& \geq \sum_{i=j}\left|\varphi\left(x_{i}\right)\right|^{2}+\sum_{i \neq j} \overline{\varphi\left(x_{i}\right)} \varphi\left(x_{j}\right) \\
& =\left|\sum_{i} \varphi\left(x_{i}\right)\right|^{2} \\
& =|\varphi(x)|^{2} .
\end{aligned}
$$

So let us consider the case of $x_{i}=\sum a^{(k)} y^{(k)}$ with all $a^{(k)} \in \mathcal{A}_{i}$. Then the second part of Lemma 2.1. gives

$$
\begin{aligned}
& \varphi\left(x_{i}^{*} x_{i}\right) \\
& =\sum_{k, l} \varphi\left(y^{(k) *} a^{(k) *} a^{(l)} y^{(l)}\right) \\
& =\sum_{k, l} \psi_{i}\left(a^{(k) *} a^{(l)}\right) \varphi\left(y^{(k) *} y^{(l)}\right)-\sum_{k, l} \psi_{i}\left(a^{(k) *} a^{(l)}\right) \varphi\left(y^{(k) *}\right) \varphi\left(y^{(l)}\right) \\
& \quad+\sum_{k, l} \varphi_{i}\left(a^{(k) *} a^{(l)}\right) \varphi\left(y^{(k) *}\right) \varphi\left(y^{(l)}\right) .
\end{aligned}
$$

By positivity of $\varphi_{i}$ and $\psi_{i}$ we can write

$$
\varphi_{i}\left(a^{(k) *} a^{(l)}\right)=\sum_{r} \overline{\alpha_{r}^{(k)}} \alpha_{r}^{(l)}, \quad \psi_{i}\left(a^{(k) *} a^{(l)}\right)=\sum_{r} \overline{\beta_{r}^{(k)}} \beta_{r}^{(l)}
$$

for some $\alpha_{r}^{(k)}, \beta_{r}^{(k)} \in \mathbb{C}$. By using our induction hypothesis for $\sum_{k} \beta_{r}^{(k)} y^{(k)}$ this implies

$$
\begin{aligned}
\sum_{k, l} \psi_{i}\left(a^{(k) *} a^{(l)}\right) \varphi\left(y^{(k) *} y^{(l)}\right) & =\sum_{k, l, r} \overline{\beta_{r}^{(k)}} \beta_{r}^{(l)} \varphi\left(y^{(k) *} y^{(l)}\right) \\
& =\sum_{r} \varphi\left[\left(\sum_{k} \beta_{r}^{(k)} y^{(k)}\right)^{*}\left(\sum_{l} \beta_{r}^{(l)} y^{(l)}\right)\right] \\
& \geq \sum_{r}\left|\varphi\left(\sum_{k} \beta_{r}^{(k)} y^{(k)}\right)\right|^{2} \\
& =\sum_{k, l} \psi_{i}\left(a^{(k) *} a^{(l)}\right) \varphi\left(y^{(k) *}\right) \varphi\left(y^{(l)}\right) .
\end{aligned}
$$

But then, again by our induction hypothesis

$$
\varphi\left(x_{i}^{*} x_{i}\right) \geq \sum_{k, l} \varphi_{i}\left(a^{(k) *} a^{(l)}\right) \varphi\left(y^{(k) *}\right) \varphi\left(y^{(l)}\right)
$$




$$
\begin{aligned}
& =\sum_{r} \sum_{k, l} \overline{\alpha_{r}^{(k)}} \alpha_{r}^{(l)} \varphi\left(y^{(k) *}\right) \varphi\left(y^{(l)}\right) \\
& =\sum_{r} \varphi\left[\left(\sum_{k} \alpha_{r}^{(k)} \varphi\left(y^{(k)}\right)\right)^{*}\left(\sum_{l} \alpha_{r}^{(l)} \varphi\left(y^{(l)}\right)\right)\right] \\
& \geq \sum_{k, l} \varphi_{i}\left(a^{(k) *}\right) \varphi_{i}\left(a^{(l)}\right) \varphi\left(y^{(k) *}\right) \varphi\left(y^{(l)}\right) \\
& =\sum_{k, l} \varphi\left(a^{(k) *} y^{(k) *}\right) \varphi\left(a^{(l)} y^{(l)}\right) \\
& =\left|\varphi\left(x_{i}\right)\right|^{2} .
\end{aligned}
$$

\section{Remarks.}

(1) If we have $\varphi_{i}=\psi_{i}$ for all $i \in I$, then we recover the case of the free product [Voi1, VDN] and we obtain an algebraic proof for the positivity also in this case, thus giving a positive answer to a question posed in [Spe2].

(2) If we want to make our construction associative, then we should extend also the $\psi_{i}$ to a new state $\psi$ on $\mathcal{A}$. It is clear that $\psi$ should be the free product of the $\psi_{i}$, in our notations $\psi:=*_{i \in I}\left(\psi_{i}, \psi_{i}\right)$. This together with $\varphi:=*_{i \in I}\left(\varphi_{i}, \psi_{i}\right)$ will be denoted by

$$
(\varphi, \psi)=*_{i \in I}\left(\varphi_{i}, \psi_{i}\right)
$$

(not to be confused with our notation of a symmetrized product in [BSp]). With these definitions one gets directly the associativity of our c-free product: If $I=I_{1} \cup I_{2}$ with $I_{1} \cap I_{2}=\emptyset$, then

$$
*_{i \in I}\left(\varphi_{i}, \psi_{i}\right)=\left\{*_{i \in I_{1}}\left(\varphi_{i}, \psi_{i}\right)\right\} *\left\{*_{i \in I_{2}}\left(\varphi_{i}, \psi_{i}\right)\right\} .
$$

(3) Commutativity of our construction is clear.

(4) Cabanal-Duvillard [CDu] introduced a generalization of our construction from two to infinitely many states. However, his product ceases to be associative.

\section{Combinatorial description of the c-free convolution.}

Let $\mathcal{M}$ be the set of all compactly supported probability measures on $\mathbb{R}$. Since such a measure $\mu$ is determined by its moments we can identify it with a state on the $*$-algebra $\mathbb{C}\langle X\rangle$ (where $X^{*}=X$ ) via

$$
\mu\left(X^{n}\right)=\int t^{n} d \mu(t) \quad(n \geq 0) .
$$


Let now $\mu_{1}, \mu_{2}, \nu_{1}, \nu_{2} \in \mathcal{M}$ be given. We identify $\mu_{i}, \nu_{i}$ with states on $\mathbb{C}\left\langle X_{i}\right\rangle(i=1,2)$ and get, by our results from Sect. 2 , the c-free product $\varphi=\left(\mu_{1}, \nu_{1}\right) *\left(\mu_{2}, \nu_{2}\right)$ on $\mathbb{C}\left\langle X_{1}\right\rangle * \mathbb{C}\left\langle X_{2}\right\rangle=\mathbb{C}\left\langle X_{1}, X_{2}\right\rangle$ (the latter being the algebra of polynomials in the non-commuting variables $X_{1}$ and $\left.X_{2}\right)$. The c-free convolution

$$
\mu=\left(\mu_{1}, \nu_{1}\right) \boxplus\left(\mu_{2}, \nu_{2}\right) \in \mathcal{M}
$$

is then given as the distribution of $X:=X_{1}+X_{2}$, i.e.

$$
\int t^{n} d \mu(t)=\mu\left(X^{n}\right)=\varphi\left(\left(X_{1}+X_{2}\right)^{n}\right) \quad(n \geq 0) .
$$

For $\mu_{i}=\nu_{i}(i=1,2)$ this reduces to the free convolution of Voiculescu [VDN].

As in Remark 2 of Sect. 2, we define also a measure $\nu$ as the free convolution of $\nu_{1}$ and $\nu_{2}$, i.e.

$$
\nu=\left(\nu_{1}, \nu_{1}\right) \boxplus\left(\nu_{2}, \nu_{2}\right)=\nu_{1} \boxplus \nu_{2},
$$

and denote this situation by

$$
(\mu, \nu)=\left(\mu_{1}, \nu_{1}\right) \boxplus\left(\mu_{2}, \nu_{2}\right)
$$

Then our mapping $\boxplus: \mathcal{M}^{2} \times \mathcal{M}^{2} \rightarrow \mathcal{M}^{2}$ is commutative and associative.

Our aim is now to extend the combinatorial description of the free convolution with the help of the lattice of non-crossing partitions [Spe2] to our case.

For a $\mu \in \mathcal{M}$ we denote its moments by

$$
m_{n}(\mu):=\mu\left(X^{n}\right)=\int t^{n} d \mu(t)
$$

and we want to understand (at least in principle) the connection between

$$
\left(m_{n}(\mu), m_{n}(\nu)\right)_{n \in \mathbb{N}} \quad \text { and } \quad\left(m_{n}\left(\mu_{1}\right), m_{n}\left(\nu_{1}\right)\right)_{n \in \mathbb{N}},\left(m_{n}\left(\mu_{2}\right), m_{n}\left(\nu_{2}\right)\right)_{n \in \mathbb{N}} \cdot
$$

As in the case of the free convolution this connection is quite complicated and it is advantageous to introduce new quantities, called cumulants, which linearize the convolution. These cumulants are connected with the notion of non-crossing partitions.

Definitions. Let $\pi=\left\{V_{1}, \ldots, V_{p}\right\}$ be a partition of the linear ordered set $\{1, \ldots, n\}$, i.e. the $V_{i} \neq \emptyset$ are ordered and disjoint sets whose union is $\{1, \ldots, n\}$. Then $\pi$ is called non-crossing if $a, c \in V_{i}$ and $b, d \in V_{j}$ with 
$a<b<c<d$ implies $i=j$.

The sets $V_{i} \in \pi$ are called blocks. A block $V_{i}$ of a non-crossing partition $\pi=\left\{V_{1}, \ldots, V_{p}\right\}$ is called inner, if there exists a $V_{j} \in \pi$ and $a, b \in V_{j}$ such that $a<v<b$ for at least one (and hence for all) $v \in V_{i}$. A block $V_{i} \in \pi$ which is not inner is called outer.

We will denote the set of all non-crossing partitions of the set $\{1, \ldots, n\}$ by $N C(n)$. By $N C_{2}(2 n)$ we denote those non-crossing partitions $\pi=\left\{V_{1}, \ldots\right.$, $\left.V_{n}\right\} \in N C(2 n)$ where each block $V_{i} \in \pi$ consists of exactly two elements.

The notion of non-crossing partition was introduced by Kreweras [Kre], the distinction between outer and inner blocks was considered in [BSp].

After this preparations we can now introduce the notion of cumulants. For the description of $\nu=\nu_{1} \boxplus \nu_{2}$ we have to use the free or non-crossing cumulants $r_{n}=r_{n}(\nu)$ (see [Spe2, NSp1, NSp2]), defined recursively in terms of the moments $m_{n}=m_{n}(\nu)$ by

$$
m_{n}=\sum_{k=1}^{n} \sum_{\substack{l(1), \ldots, l(k) \geq 0 \\ l(1)+\cdots+l(k)=n-k}} r_{k} m_{l(1)} \ldots m_{l(k)} .
$$

This definition may be indicated symbolically by

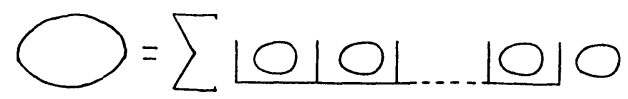

and it is equivalent to

$$
m_{n}=\sum_{\substack{\pi=\left\{V_{1}, \ldots, V_{p}\right\} \\ \in N C(n)}} r_{V_{1}} \ldots r_{V_{p}}=\sum_{\pi \in N C(n)} \prod_{V_{l} \in \pi} r_{V_{l}}
$$

or

$$
r_{n}=\sum_{\substack{\pi=\left\{V_{1}, \ldots, V_{p}\right\} \\ \in N C(n)}} m_{V_{1}} \ldots m_{V_{p}} \cdot \mu\left(\pi, 1_{n}\right)=\sum_{\pi \in N C(n)} \mu\left(\pi, 1_{n}\right) \prod_{V_{l} \in \pi} m_{V_{l}}
$$

where we have used the notation $m_{V}:=m_{|V|}$ and $r_{V}:=r_{|V|}$ for some set $V$ (with $|V|$ being the number of elements in $V$ ). The function $\mu\left(\pi, 1_{n}\right)$ is the Möbius function of the lattice of non-crossing partitions and is just determined by resolving (1) for the $r_{n}$ in terms of the $m_{i}$. 
Free convolution is then described by [Voi2, Spe2]

$$
r_{n}\left(\nu_{1} \boxplus \nu_{2}\right)=r_{n}\left(\nu_{1}\right)+r_{n}\left(\nu_{2}\right) \quad \text { for all } n \geq 1 .
$$

For our c-free convolution we have, for a given pair $(\mu, \nu)$, to introduce, in addition to $r_{n}=r_{n}(\nu)$, also c-free cumulants $R_{n}=R_{n}(\mu, \nu)$, which do not only depend on the moments of $\mu$ but also on those of $\nu$. The most instructive definition is again by recursion, namely

$$
m_{n}(\mu)=\sum_{k=1}^{n} \sum_{\substack{l(1), \ldots, l(k) \geq 0 \\ l(1)+\cdots+l(k)=n-k}} R_{k} \cdot m_{l(1)}(\nu) \cdot \ldots \cdot m_{l(k-1)}(\nu) \cdot m_{l(k)}(\mu),
$$

pictorially

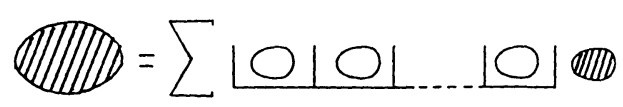

Note that the "inner" moments are given by $\nu$, only the "outer" one is connected with $\mu$. Of course, the free cumulants are recovered from this by $r_{n}(\nu)=R_{n}(\nu, \nu)$.

The above definition is equivalent to a generalization of (1), namely

$$
m_{n}(\mu)=\sum_{\pi \in N C(n)} \prod_{\substack{V_{l} \in \pi \\ V_{l} \text { inner }}} r_{V_{l}} \prod_{\substack{V_{k} \in \pi \\ V_{k} \text { outer }}} R_{V_{k}} .
$$

The following example shows that the analogue of formula (2) is not true for the c-free cumulants.

Example. We have

$$
\begin{aligned}
& m_{3}(\nu)=r_{3}(\nu)+2 r_{2}(\nu) \cdot r_{1}(\nu)+r_{1}(\nu)^{3}+r_{2}(\nu) \cdot r_{1}(\nu) \\
& m_{3}(\mu)=R_{3}(\mu, \nu)+2 R_{2}(\mu, \nu) \cdot R_{1}(\mu, \nu)+R_{1}(\mu, \nu)^{3}+R_{2}(\mu, \nu) \cdot r_{1}(\nu),
\end{aligned}
$$

but

$$
\begin{aligned}
r_{3}(\nu)= & m_{3}(\nu)-2 m_{2}(\nu) \cdot m_{1}(\nu)-m_{2}(\nu) \cdot m_{1}(\nu)+2 m_{1}(\nu)^{3} \\
R_{3}(\mu, \nu)= & m_{3}(\mu)-2 m_{2}(\mu) \cdot m_{1}(\mu)-m_{2}(\mu) \cdot m_{1}(\nu)+m_{1}(\mu)^{3} \\
& +m_{1}(\mu)^{2} \cdot m_{1}(\nu) .
\end{aligned}
$$

But nevertheless we have the following theorem.

Theorem 3.1. The c-free convolution

$$
(\mu, \nu)=\left(\mu_{1}, \nu_{1}\right) \boxplus\left(\mu_{2}, \nu_{2}\right)
$$


is described by

$$
r_{n}(\nu)=r_{n}\left(\nu_{1}\right)+r_{n}\left(\nu_{2}\right)
$$

and

$$
R_{n}(\mu, \nu)=R_{n}\left(\mu_{1}, \nu_{1}\right)+R_{n}\left(\mu_{2}, \nu_{2}\right)
$$

for all $n \geq 1$.

Proof. The proof follows the same line of arguing as in [Spe2]. Given $(\varphi, \psi)$ on some unital $*$-algebra $\mathcal{A}$, we define more general cumulant functions $r=$ $\left(r_{n}\right)$ and $R=\left(R_{n}\right)$ with

$$
r_{n}, R_{n}: \underbrace{\mathcal{A} \times \cdots \times \mathcal{A}}_{n \text {-times }} \rightarrow \mathbb{C} \quad(n \geq 1)
$$

by

$$
\begin{aligned}
\psi\left(a_{1} \ldots a_{n}\right)= & \sum_{k=0}^{n-1} \sum_{1<l(1)<\cdots<l(k) \leq n} r_{k+1}\left[a_{1}, a_{l(1)}, \ldots, a_{l(k)}\right] \\
& \cdot \psi\left(a_{2} \ldots a_{l(1)-1}\right) \ldots \psi\left(a_{l(k-1)+1} \ldots a_{l(k)-1}\right) \psi\left(a_{l(k)+1} \ldots a_{n}\right)
\end{aligned}
$$

and

(B)

$$
\begin{aligned}
\varphi\left(a_{1} \ldots a_{n}\right)= & \sum_{k=0}^{n-1} \sum_{1<l(1)<\cdots<l(k) \leq n} R_{k+1}\left[a_{1}, a_{l(1)}, \ldots, a_{l(k)}\right] \\
& \cdot \psi\left(a_{2} \ldots a_{l(1)-1}\right) \ldots \psi\left(a_{l(k-1)+1} \ldots a_{l(k)-1}\right) \varphi\left(a_{l(k)+1} \ldots a_{n}\right)
\end{aligned}
$$

for all $a_{1}, \ldots, a_{n} \in \mathcal{A}$. These equations can recursively be resolved for the definition of $r_{n}\left[a_{1}, \ldots, a_{n}\right]$ and $R_{n}\left[a_{1}, \ldots, a_{n}\right]$.

Let now $\left(\mu_{i}, \nu_{i}\right)$ on $\mathcal{A}_{i}=\mathbb{C}\left\langle X_{i}\right\rangle$ for $i=1,2$ be given. Then we obtain in the above way the functions $r\left(\mu_{i}\right)$ and $R\left(\mu_{i}, \nu_{i}\right)$ on $\bigcup_{n=1}^{\infty} \mathcal{A}_{i}^{\times n}$. On $\bigcup_{n=1}^{\infty}\left(\mathcal{A}_{1} \cup\right.$ $\left.\mathcal{A}_{2}\right)^{\times n} \subset \bigcup_{n=1}^{\infty}\left(\mathcal{A}_{1} * \mathcal{A}_{2}\right)^{\times n}$ we define their direct sum

$$
r:=r\left(\mu_{1}\right) \oplus r\left(\mu_{2}\right) \quad \text { and } \quad R:=R\left(\mu_{1}, \nu_{1}\right) \oplus R\left(\mu_{2}, \nu_{2}\right)
$$

by

$$
r_{n}\left[a_{1}, \ldots, a_{n}\right]= \begin{cases}r_{n}\left(\mu_{1}\right)\left[a_{1}, \ldots, a_{n}\right], & \text { if all } a_{i} \in \mathcal{A}_{1} \\ r_{n}\left(\mu_{2}\right)\left[a_{1}, \ldots, a_{n}\right], & \text { if all } a_{i} \in \mathcal{A}_{2} \\ 0, & \text { otherwise }\end{cases}
$$


and

$$
R_{n}\left[a_{1}, \ldots, a_{n}\right]= \begin{cases}R_{n}\left(\mu_{1}, \nu_{1}\right)\left[a_{1}, \ldots, a_{n}\right], & \text { if all } a_{i} \in \mathcal{A}_{1} \\ R_{n}\left(\mu_{2}, \nu_{2}\right)\left[a_{1}, \ldots, a_{n}\right], & \text { if all } a_{i} \in \mathcal{A}_{2} \\ 0, & \text { otherwise }\end{cases}
$$

for all $a_{1}, \ldots, a_{n} \in \mathcal{A}_{1} \cup \mathcal{A}_{2} \subset \mathcal{A}_{1} * \mathcal{A}_{2}$. Note that there is no ambiguity in this definition because in the case that some $a_{i} \in \mathcal{A}_{1} \cap \mathcal{A}_{2}=\mathbb{C} 1$, both values, $R_{n}\left(\mu_{1}, \nu_{1}\right)$ and $R_{n}\left(\mu_{2}, \nu_{2}\right)$, are the same.

Now we use the recursion formulas $(A)$ and $(B)$ for the definition of the states $\psi$ and $\varphi$ on $\mathcal{A}=\mathcal{A}_{1} * \mathcal{A}_{2}$. One has to check that this is well-defined because there are different possibilities for writing elements $a \in \mathcal{A}$ as sums of products $a_{1} \ldots a_{n}$ with $a_{1}, \ldots, a_{n} \in \mathcal{A}_{1} \cup \mathcal{A}_{2}$. But since this ambiguity comes only from relations inside $\mathcal{A}_{1}$ and relations inside $\mathcal{A}_{2}$, which are respected by $r$ and $R$ (because they are respected by $r\left(\nu_{i}\right)$ and $R\left(\mu_{i}, \nu_{i}\right)$ ), no problem occurs; for more details on this, see [Spe2].

It only remains to see that $(\varphi, \psi)$ on $\mathcal{A}=\mathbb{C}\left\langle X_{1}, X_{2}\right\rangle$ is indeed the c-free product of $\left(\mu_{1}, \nu_{1}\right)$ and $\left(\mu_{2}, \nu_{2}\right)$, i.e. we have to check that it fulfills the characterizing property of the c-free product. For $\psi$ this follows from the results of [Spe2]. So consider $a \in \mathcal{A}$ of the form $a=a_{1} \ldots a_{n}$ with $a_{j} \in \mathcal{A}_{i(j)}$,

$$
i(1) \neq i(2) \neq \cdots \neq i(n), \quad \nu_{i(j)}\left(a_{j}\right)=0 .
$$

Note that in $(B)$, because of the definition of $R$ and the fact that $\psi$ is the free product of $\psi_{1}$ and $\psi_{2}$, only the term with $k=0$ survives, i.e.

$$
\varphi\left(a_{1} \ldots a_{n}\right)=R_{1}\left[a_{1}\right] \cdot \varphi\left(a_{2} \ldots a_{n}\right)=\varphi_{i(1)}\left(a_{1}\right) \cdot \varphi\left(a_{2} \ldots a_{n}\right),
$$

which gives, by induction, the wanted factorization for $\varphi$.

To get the assertion of the theorem, one has now to use the definition of $R$ as the direct sum of $R\left(\mu_{1}, \nu_{1}\right)$ and $R\left(\mu_{2}, \nu_{2}\right)$

$$
\begin{aligned}
R_{n}(\mu, \nu) & \hat{=} R_{n}\left[X_{1}+X_{2}, \ldots, X_{1}+X_{2}\right] \\
& =R_{n}\left[X_{1}, \ldots, X_{1}\right]+R_{n}\left[X_{2}, \ldots, X_{2}\right] \\
& \hat{=} R_{n}\left(\mu_{1}, \nu_{1}\right)+R_{n}\left(\mu_{2}, \nu_{2}\right),
\end{aligned}
$$

and the same for $r$.

\section{Remarks.}

(1) The description of the c-free convolution in terms of cumulants can, analogously to the free case [Spe2], be generalized to a description of 
the c-free product. Indeed, in our proof we had to use the corresponding machinery for the c-free product on $\mathbb{C}\left\langle X_{1}, X_{2}\right\rangle=\mathbb{C}\left\langle X_{1}\right\rangle * \mathbb{C}\left\langle X_{2}\right\rangle$.

(2) An interesting special case of the c-free convolution is given if we put $\nu_{i}=\delta_{0}$. Then only outer sets survive in the definition of the c-free cumulants. This leads to a description in terms of interval partitions, which were introduced by von Waldenfels $[\mathbf{v W a}$ ]. The corresponding convolution $\left(\mu, \delta_{0}\right)=\left(\mu_{1}, \delta_{0}\right) \boxplus\left(\mu_{2}, \delta_{0}\right)$ shares a lot of properties with the usual and the free convolution. This "boolean" convolution was investigated in [Wor], the results will be published in [SpW].

\section{Limit theorems for the c-free convolution.}

To become familiar with the connection between non-crossing partitions and the c-free convolution, we will now calculate quite explicitly the c-free central and Poisson limit distribution. A more systematic machinery for the treatment of such questions will be presented in the next section.

We will see (comp. [BSp]) that the moments of the limit distributions are calculated with the help of the partitions in $N_{2}(2 n)$ or $N C(n)$. Thus, before presenting the limit theorems, we collect all relevant information on the combinatorics of the respective partitions in two lemmas. These combinatorial statements have also some interest of their own. Although there has been an increasing interest in the lattice of non-crossing partitions in the last time [Ede1, Ede2, Pou, Sim, SiU, Bia, Nic], we have not found any investigation on this subject related to the distinction between "inner" and "outer".

First, for the central limit theorem, we have to consider $N C_{2}(2 n)$. We will need the numbers $(n \in \mathbb{N}, 0 \leq k \leq n)$

$$
c^{n}:=\# N C_{2}(2 n)
$$

$a_{k}^{n}:=\#\left\{\pi \in N C_{2}(2 n) \mid\right.$ the number of inner sets of $\pi$ is equal to $\left.k\right\}$.

Of course, we have $a_{n}^{n}=0$.

For the investigation of these quantities it is advantageous to use the well known bijection between partitions $\pi \in N C_{2}(2 n)$ and $n$-Catalan paths $\Lambda$ (see, e.g., $[\mathbf{H i P}]$ ). An $n$-Catalan path $\Lambda \hat{=}\left\{s_{1}, \ldots, s_{2 n}\right\}$ is a graph in $\mathbb{Z}^{2}$, starting at $(0,0)$, ending at $(n, n)$, with possible steps $s_{i}=(0,1)$ or $s_{i}=(1,0)$ $(i=1, \ldots, 2 n)$, such that no part of the path lies above the diagonal. The above bijection is given as follows: To each $\pi=\left\{V_{1}, \ldots, V_{n}\right\} \in N C_{2}(2 n)$ we assign a $\Lambda(\pi) \hat{=}\left\{s_{1}, \ldots, s_{2 n}\right\}$ in the way that $s_{i}=(1,0)$ if $i$ is the first element in one of the $V_{j}$, and $s_{i}=(0,1)$ if $i$ is the second element in one of the $V_{j}$. The number of outer sets of $\pi$ corresponds thereby to the number of points $(i, i)(1 \leq i \leq n)$, where $\Lambda(\pi)$ meets the diagonal. 
Example. For $\pi=\{(1,4),(2,3),(5,6)\}$ we have

$$
\Lambda(\pi)=\{(1,0),(1,0),(0,1),(0,1),(1,0),(0,1)\}
$$

which corresponds to the following graph:

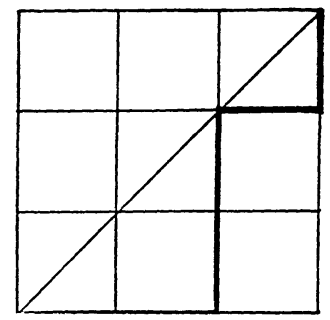

It is a well known fact [HiP] that the number of all $n$-Catalan paths is given by the

$$
\text { Catalan number } \quad c_{n}:=\frac{1}{n}\left(\begin{array}{c}
2 n \\
n-1
\end{array}\right),
$$

hence

$$
c^{n}=c_{n}=\frac{1}{n}\left(\begin{array}{c}
2 n \\
n-1
\end{array}\right)=\frac{1}{n+1}\left(\begin{array}{c}
2 n \\
n
\end{array}\right) .
$$

This follows quite easily from the recursion formula

$$
c^{n}=\sum_{k=1}^{n} c^{k-1} c^{n-k}, \quad \text { where } c^{0}:=1
$$

which is the recursion for the Catalan numbers.

It seems that $a_{k}^{n}$ has not received any interest so far. We collect their basic properties in the next lemma.

\section{Lemma 4.1.}

(i) We have for $n \geq 1$

$$
a_{0}^{n}=1
$$

(ii) We have for $n \geq 2$

$$
a_{n-1}^{n}=a_{n-2}^{n}=c^{n-1}=\frac{1}{n}\left(\begin{array}{c}
2 n-2 \\
n-1
\end{array}\right) .
$$

(iii) We have for $n \geq 2$ and $0 \leq k \leq n-2$

$$
a_{k}^{n}+a_{k+1}^{n-1}=a_{k+1}^{n} .
$$


Proof. (i) There is only one $n$-Catalan path which meets the diagonal $n$ times, namely $\Lambda \triangleq\{(1,0),(0,1),(1,0),(0,1), \ldots\}$.

(ii) Shifting the diagonal one unit to the right induces a bijection between the set of all $n$-Catalan paths which meet the diagonal once and the set of all $(n-1)$-Catalan paths. Hence $a_{n-1}^{n}=c^{n-1}$.

For $a_{n-2}^{n}$ we have, denoting with $(k, k)$ the first intersection point with the diagonal,

$$
a_{n-2}^{n}=\sum_{k=1}^{n-1} a_{k-1}^{k} a_{n-k-1}^{n-k}=\sum_{k=1}^{n-1} c^{k-1} c^{n-k-1}=c^{n-1},
$$

by the recursion formula for the Catalan numbers.

(iii) We prove this by induction on $n$. For $n=2$, the assertion is true, namely for $k=0$ we have

$$
a_{0}^{2}+a_{1}^{1}=1+0=1=a_{1}^{2} .
$$

Now assume the assertion to be true for all $n^{\prime}$ with $2 \leq n^{\prime}<n$. We want to show it for $n$.

First, consider $k$ with $0 \leq k \leq n-4$. Again we use the general decomposition

$$
a_{k+1}^{n}=\sum_{l=1}^{k+2} a_{l-1}^{l} a_{k-l+2}^{n-l}
$$

which results from the splitting of an $n$-Catalan path into two parts, the first one from $(0,0)$ to its first intersection point $(l, l)$ with the diagonal (this part thus gives rise to $l-1$ inner sets) and the remaining $(n-l)$-Catalan path, which has to produce the remaining $(k+1)-(l-1)$ inner sets. The decomposition $(*)$ is true for all $n \geq 2$ and $k$ with $0 \leq k \leq n-3$. Since $0 \leq k \leq n-4$, we have $0 \leq k-l+1 \leq n-l-3 \leq n-l-2$ and, for all $l$ with $1 \leq l \leq k+1$, we can use our induction hypothesis for $n^{\prime}=n-l$ to obtain

$$
\begin{aligned}
a_{k+1}^{n} & =\sum_{l=1}^{k+1} a_{l-1}^{l} a_{(k-l+1)+1}^{n-l}+a_{k+1}^{k+2} \cdot 1 \\
& =\sum_{l=1}^{k+1} a_{l-1}^{l}\left(a_{k-l+1}^{n-l}+a_{k-l+2}^{n-l-1}\right)+a_{k+1}^{k+2} \cdot 1 \\
& =\sum_{l=1}^{k+1} a_{l-1}^{l} a_{k-l+1}^{n-l}+\sum_{l=1}^{k+2} a_{l-1}^{l} a_{k-l+2}^{n-l-1}
\end{aligned}
$$




$$
=a_{k}^{n}+a_{k+1}^{n-1},
$$

the last equality again by application of $(*)$.

Now consider $k=n-3$. Then the same arguments as before apply, but now $a_{k-l+2}^{n-l-1}=0$ for $l=1, \ldots, k+1$, thus

$$
a_{k+1}^{n}=\sum_{l=1}^{k+1} a_{l-1}^{l} a_{k-l+1}^{n-l}+a_{k+1}^{k+2}=a_{k}^{n}+a_{k+1}^{n-1} .
$$

For $k=n-2$, the assertion reduces to ii), because $a_{k+1}^{n-1}=a_{n-1}^{n-1}=0$.

For the treatment of the c-free Poisson distribution we will need some specific information on the combinatorics of the sets $N C(n)$, namely we will use $(n \geq 1,1 \leq k \leq n, 0 \leq l \leq n-1)$

$t_{k}^{n}:=\#\{\pi \in N C(n) \mid \pi$ consists precisely of $k$ sets $\}$

$s_{k, l}^{n}:=\#\{\pi \in N C(n) \mid \pi$ consists precisely of $k$ outer and $l$ inner sets $\}$.

In addition, we define $t_{0}^{n}:=0$ for $n \geq 1$ and $t_{0}^{0}:=1$. Similarly, we put $s_{k, l}^{n}:=0$ if the indices are out of their natural domain, with the only exception $s_{0,0}^{0}:=1$.

\section{Lemma 4.2.}

(i) We have for $n \geq 1$ and $1 \leq k \leq n$

$$
t_{k}^{n}=t_{k-1}^{n-1}+\sum_{r=2}^{n} \sum_{i=1}^{r-1} t_{i}^{r-1} t_{k-i}^{n-r} .
$$

(ii) We have for $n \geq 2$ and $0 \leq l \leq n-1$

$$
s_{1, l}^{n}=t_{l+1}^{n-1} .
$$

(iii) We have for $n \geq 1$ and $k, l \geq 0$

$$
s_{k+1, l}^{n}=\sum_{r=1}^{n} \sum_{j=0}^{l} s_{1, j}^{r} s_{k, l-j}^{n-r} .
$$

Proof. (i) Let $\pi=\left\{V_{1}, \ldots, V_{k}\right\} \in N C(n)$ consist of $k$ sets, with $1 \in V_{1}$. Then there are two disjoint possibilities: either $V_{1}=(1)$ or $V_{1} \neq(1)$. In the first case, $\pi \mapsto \pi \backslash(1)$ gives a bijection onto all non-crossing partitions of $\{2, \ldots, n\}$ consisting of $k-1$ sets. In the second case, let $r \neq 1$ be the maximal element of $V_{1}$. Then, removing $r$ from $V_{1}, \pi$ splits into a non-crossing partition of 
$\{1, \ldots, r-1\}$ (which may consist of $i$ sets, where possibly $1 \leq i \leq r-1$ ) and a non-crossing partition of $\{r+1, \ldots, n\}$ (which has to consist of the remaining $k-i$ sets). If $r=n$, then $k=i$, and we need the special definition $t_{0}^{0}:=1$. The formula is also true for $n=1$, since then $t_{1}^{1}=t_{0}^{0}=1$.

(ii) If $\pi \in N C(n)$ has $l$ inner sets and only one outer set $V_{1}$, then $1, n \in$ $V_{1}$ and the removing of $n(n \neq 1)$ gives a bijection onto all non-crossing partitions of $\{1, \ldots, n-1\}$ consisting of $l+1$ sets.

(iii) Let $r$ be the maximal element of the first set $V_{1}$ in $\pi \in N C(n)$. Then $\pi$ decomposes into a non-crossing partition of $\{1, \ldots, r\}$ with $V_{1}$ as the only outer set (and possibly $j$ inner sets) and a non-crossing partition of $\{r+1, \ldots, n\}$ which has to yield the remaining $k$ outer and $l-j$ inner sets. If $k=0$ and $r=n$, then $j=0$, and we need $s_{0,0}^{0}=1$.

Remark. Kreweras [Kre] gives the following explicit formula for $t_{k}^{n}$

$$
t_{k}^{n}=\frac{(n-1) ! n !}{(k-1) ! k !(n-k) !(n-k+1) !},
$$

but for our investigations the recurrence formula of our lemma is much more useful.

Now we have finished the presentation of all needed combinatorial tools and we can start our investigations on limit theorems for the c-free convolution.

Let us denote, for $\lambda>0$, by $D_{\lambda}$ the dilation of probability measures on $\mathbb{R}$ by the factor $\lambda$, i.e.

$$
\left(D_{\lambda} \mu\right)(A):=\mu\left(\lambda^{-1} A\right) \quad \text { for } A \subset \mathbb{R} \text { measurable, }
$$

and

$$
D_{\lambda}(\mu, \nu):=\left(D_{\lambda} \mu, D_{\lambda} \nu\right)
$$

Under the weak convergence

$$
\underset{N \rightarrow \infty}{\mathrm{w}-\lim }\left(\mu_{N}, \nu_{N}\right)=(\mu, \nu)
$$

we will understand the componentwise weak convergence

$$
\underset{N \rightarrow \infty}{\mathrm{w}}-\lim _{N} \mu_{N}=\mu \quad \text { and } \quad \underset{N \rightarrow \infty}{\mathrm{w}}-\lim _{N} \nu_{N}=\nu \text {. }
$$

Theorem 4.3 (c-free central limit theorem). Let $(\mu, \nu) \in \mathcal{M}^{2}$ with

$$
\mu(X)=\nu(X)=0 \quad \text { and } \quad \mu\left(X^{2}\right)=\alpha^{2}, \quad \nu\left(X^{2}\right)=\beta^{2} \quad(\alpha, \beta \geq 0)
$$


be given. Then we have

$$
\underset{N \rightarrow \infty}{\mathrm{w}-\lim _{\sqrt{1 / N}}}\{\underbrace{(\mu, \nu) \boxplus \ldots \boxplus(\mu, \nu)}_{N \text {-times }}\}=\left(\nu_{\alpha, \beta}, \nu_{\beta, \beta}\right),
$$

where

$$
\nu_{\alpha, \beta}=c\left(\delta_{\alpha^{2} / \sqrt{\alpha^{2}-\beta^{2}}}+\delta_{-\alpha^{2} / \sqrt{\alpha^{2}-\beta^{2}}}\right)+\tilde{\nu}_{\alpha, \beta}
$$

with

$$
\begin{gathered}
c= \begin{cases}\frac{1}{4} \frac{\alpha^{2}-2 \beta^{2}}{\alpha^{2}-\beta^{2}}, & 0 \leq \frac{\beta^{2}}{\alpha^{2}} \leq \frac{1}{2} \\
0, & \frac{1}{2} \leq \frac{\beta^{2}}{\alpha^{2}}\end{cases} \\
d \tilde{\nu}_{\alpha, \beta}(t)=\chi_{[-2 \beta, 2 \beta]}(t) \frac{1}{2 \pi} \frac{\alpha^{2} \sqrt{4 \beta^{2}-t^{2}}}{\alpha^{4}-\left(\alpha^{2}-\beta^{2}\right) t^{2}} d t .
\end{gathered}
$$

In particular

$$
d \nu_{\beta, \beta}(t)=\chi_{[-2 \beta, 2 \beta]}(t) \frac{1}{2 \pi \beta^{2}} \sqrt{4 \beta^{2}-t^{2}} d t
$$

Remark. Of course, the statement about the convergence of the second component is nothing else but the free central limit theorem [Voi2, VDN], [Spe1, Maa, Gir].

Proof. Since $\nu_{\alpha, \beta}$ and $\nu_{\beta, \beta}$ have compact support, it suffices to check that the moments of $D_{\sqrt{1 / N}}\{(\mu, \nu) \boxplus \ldots \boxplus(\mu, \nu)\}$ converge to the corresponding moments of $\left(\nu_{\alpha, \beta}, \nu_{\beta, \beta}\right)$. Note that

$$
r_{n}\left(D_{\lambda} \nu\right)=\lambda^{n} r_{n}(\nu) \quad \text { and } \quad R_{n}\left(D_{\lambda} \mu, D_{\lambda} \nu\right)=\lambda^{n} R_{n}(\mu, \nu)
$$

for all $n \geq 0$. This shows that the limiting measures $(\hat{\mu}, \hat{\nu})$ are determined by

$$
\begin{aligned}
r_{n}(\hat{\nu}) & = \begin{cases}0, & n \neq 2 \\
r_{2}(\nu)=\beta^{2}, & n=2\end{cases} \\
R_{n}(\hat{\mu}, \hat{\nu}) & = \begin{cases}0, & n \neq 2 \\
R_{2}(\mu, \nu)=\alpha^{2}, & n=2,\end{cases}
\end{aligned}
$$


or in terms of their moments

$$
\begin{aligned}
& m_{l}(\hat{\nu})= \begin{cases}0, & l \text { odd } \\
c^{n} \beta^{2 n}, & l=2 n\end{cases} \\
& m_{l}(\hat{\mu})= \begin{cases}0, & l \text { odd } \\
\sum_{k=0}^{n-1} a_{k}^{n} \alpha^{2(n-k)} \beta^{2 k}, & l=2 n .\end{cases}
\end{aligned}
$$

This formula for the moments of $\hat{\mu}$ was also derived in [BSp].

Now consider the generating power series

$$
f(z):=\sum_{n=0}^{\infty} m_{2 n}(\hat{\nu}) z^{2 n}, \quad F(z):=\sum_{n=0}^{\infty} m_{2 n}(\hat{\mu}) z^{2 n} .
$$

The recursion formula for the Catalan numbers yields [Spe2, VDN]

$$
\beta^{2} z^{2} f(z)^{2}=f(z)-1, \text { thus } f(z)=\frac{1-\sqrt{1-4 \beta^{2} z^{2}}}{2 \beta^{2} z^{2}} .
$$

For the determination of $F(z)$, we use part (iii) of Lemma 4.1 to observe

$$
\begin{aligned}
& \left(\alpha^{2}-\beta^{2}\right) m_{2(n+1)}(\hat{\mu}) \\
& =\sum_{k=0}^{n} a_{k}^{n+1} \alpha^{2(n+2-k)} \beta^{2 k}-\sum_{k=0}^{n} a_{k}^{n+1} \alpha^{2(n+1-k)} \beta^{2(k+1)} \\
& =\sum_{k=0}^{n-1}\left(a_{k+1}^{n+1}-a_{k}^{n+1}\right) \alpha^{2(n+1-k)} \beta^{2(k+1)}+a_{0}^{n+1} \alpha^{2(n+2)}-a_{n}^{n+1} \alpha^{2} \beta^{2(n+1)} \\
& =\sum_{k=0}^{n-1} a_{k+1}^{n} \alpha^{2(n+1-k)} \beta^{2(k+1)}+\alpha^{2(n+2)}-a_{n}^{n+1} \alpha^{2} \beta^{2(n+1)} \\
& =\sum_{k=0}^{n} a_{k}^{n} \alpha^{2(n+2-k)} \beta^{2 k}-\alpha^{2} \beta^{2(n+1)} a_{n}^{n+1} \\
& =\alpha^{4} m_{2 n}(\hat{\mu})-\alpha^{2} \beta^{2(n+1)} c^{n}
\end{aligned}
$$

which implies

$$
\begin{aligned}
& \left(\alpha^{2}-\beta^{2}\right) F(z) \\
& =\left(\alpha^{2}-\beta^{2}\right)+\sum_{n=0}^{\infty} m_{2(n+1)}(\hat{\mu}) z^{2(n+1)} \\
& =\left(\alpha^{2}-\beta^{2}\right)+\alpha^{4} z^{2} \sum_{n=0}^{\infty} m_{2 n}(\hat{\mu}) z^{2 n}-\alpha^{2} \beta^{2} z^{2} \sum_{n=0}^{\infty} c^{n}(\beta z)^{2 n}
\end{aligned}
$$




$$
=\left(\alpha^{2}-\beta^{2}\right)+\alpha^{4} z^{2} F(z)-\alpha^{2} \beta^{2} z^{2} f(z) .
$$

This can be resolved for $F(z)$,

$$
F(z)=\frac{\left(\alpha^{2}-\beta^{2}\right)-\alpha^{2} \beta^{2} z^{2} f(z)}{\left(\alpha^{2}-\beta^{2}\right)-\alpha^{4} z^{2}}=\frac{\left(\alpha^{2}-\beta^{2}\right)-\frac{1}{2} \alpha^{2}\left(1-\sqrt{1-4 \beta^{2} z^{2}}\right)}{\left(\alpha^{2}-\beta^{2}\right)-\alpha^{4} z^{2}} .
$$

In terms of the Cauchy-transform $G(z)$ of $\hat{\mu}$ this reads

$$
G(z)=\frac{1}{z} F\left(\frac{1}{z}\right)=\frac{z\left(\frac{1}{2} \alpha^{2}-\beta^{2}\right)+\frac{1}{2} \alpha^{2} \sqrt{z^{2}-4 \beta^{2}}}{z^{2}\left(\alpha^{2}-\beta^{2}\right)-\alpha^{4}} .
$$

The Stieltjes inversion formula (see, e.g., [AGl]) gives then the distribution as stated in the theorem.

\section{Remarks.}

(1) An instructive way to write the Cauchy-transforms

$$
g(z)=1 / z f(1 / z) \text { and } G(z)=1 / z F(1 / z)
$$

of $\nu_{\beta, \beta}$ and $\nu_{\alpha, \beta}$, respectively, are the following continued fraction expressions

$$
\begin{gathered}
g(z)=\frac{1}{z-\frac{\beta^{2}}{z-\frac{\beta^{2}}{z-\frac{\beta^{2}}{z-\cdots}}}}, \\
G(z)=\frac{1}{z-\alpha^{2} g(z)}=\frac{1}{z-\frac{\alpha^{2}}{z-\frac{\beta^{2}}{z-\frac{\beta^{2}}{z-\cdots}}}} .
\end{gathered}
$$

These expansions follow directly from the relations

$$
\begin{aligned}
\beta^{2} z^{2} f(z) f(z) & =f(z)-1 \\
\alpha^{2} z^{2} F(z) f(z) & =F(z)-1
\end{aligned}
$$

The second identity can be checked with our explicit form of $f$ and $\tilde{F}$ or it may be derived directly by the recursion formula

$$
m_{2 n}(\hat{\mu})=\sum_{k=1}^{n} \alpha^{2} m_{2(k-1)}(\hat{\nu}) m_{2(n-k)}(\hat{\mu}) .
$$


(2) The sequence of orthogonal polynomials corresponding to $\nu_{\alpha, \beta}$ satisfies the following recurrence relations:

$$
\begin{aligned}
p_{0}(x) & =1 \\
p_{1}(x) & =x \\
p_{2}(x) & =x^{2}-\alpha^{2} \\
p_{n+1}(x) & =x p_{n}(x)-\beta^{2} p_{n-1}(x) \quad(n \geq 2) .
\end{aligned}
$$

For $\alpha^{2}=\beta^{2}=1$ we obtain the Tchebyscheff polynomials of the second kind, whereas for $\alpha^{2}=1$ and $\beta^{2}=1 / 2$ we get the Tchebyscheff polynomials of the first kind.

(3) It may be interesting to note that in the limit $\alpha, \beta \rightarrow \infty$ under the restriction $\beta / \alpha^{2}=$ const $=\gamma$, the distribution $\nu_{\alpha, \beta}$ converges to the Cauchy distribution $\mu$ with density

$$
d \mu(t)=\frac{1}{\pi} \frac{\gamma}{1+\gamma^{2} t^{2}} d t
$$

(4) In Fig. 1, we have plotted the density of $\nu_{\alpha, \beta}$ for fixed $\beta=1$ and for six different values of $\alpha$.

Theorem 4.4 (c-free Poisson limit theorem). For $\alpha, \beta \geq 0$ define for all $N \geq 1$

$$
\mu_{N}:=\left(1-\frac{\alpha}{N}\right) \delta_{0}+\frac{\alpha}{N} \delta_{1} \quad \text { and } \quad \nu_{N}:=\left(1-\frac{\beta}{N}\right) \delta_{0}+\frac{\beta}{N} \delta_{1} .
$$

Then we have

$$
\underset{N \rightarrow \infty}{\mathrm{W}-\lim }\{\underbrace{\left(\mu_{N}, \nu_{N}\right) \boxplus \ldots \boxplus\left(\mu_{N}, \nu_{N}\right)}_{N \text {-times }}\}=\left(\pi_{\alpha, \beta}, \pi_{\beta, \beta}\right),
$$

where

$$
\pi_{\alpha, \beta}=a \delta_{0}+b \delta_{z_{0}}+\tilde{\pi}_{\alpha, \beta}
$$

with

$$
\begin{aligned}
z_{0} & =\alpha+\frac{\alpha}{\alpha-\beta} \\
a & = \begin{cases}\frac{1-\beta}{1+\alpha-\beta}, & 0 \leq \beta \leq 1 \\
0, & 1 \leq \beta\end{cases} \\
b & = \begin{cases}\frac{\beta z_{0}-\alpha^{2}}{z_{0}(\beta-\alpha)}, & \alpha \leq \beta-\sqrt{\beta} \text { or } \beta+\sqrt{\beta} \leq \alpha \\
0, & \beta-\sqrt{\beta} \leq \alpha \leq \beta+\sqrt{\beta}\end{cases}
\end{aligned}
$$


and

$$
d \tilde{\pi}_{\alpha, \beta}(t)=\chi_{\left[(1-\sqrt{\beta})^{2},(1+\sqrt{\beta})^{2}\right]}(t) \frac{1}{\pi} \frac{\alpha \sqrt{4 \beta-(t-(1+\beta))^{2}}}{2 t[t(\beta-\alpha)+\alpha(1-\beta+\alpha)]} d t .
$$

In particular

$$
\begin{aligned}
\pi_{\beta, \beta} & = \begin{cases}(1-\beta) \delta_{0}+\tilde{\pi}_{\beta, \beta}, & 0 \leq \beta \leq 1 \\
\tilde{\pi}_{\beta, \beta}, & 1 \leq \beta\end{cases} \\
d \tilde{\pi}_{\beta, \beta}(t) & =\chi_{\left[(1-\sqrt{\beta})^{2},(1+\sqrt{\beta})^{2}\right]}(t) \frac{1}{2 \pi t} \sqrt{4 \beta-(t-(1+\beta))^{2}} d t .
\end{aligned}
$$

Remark. Again, the statement about the second component reduces to the free Poisson limit theorem [Maa, VDN, Spe1, Gir].

Proof. Again, it is sufficient to check the convergence of all moments. Since for $n \geq 1$

$$
m_{n}\left(\nu_{N}\right)=\frac{\beta}{N} \quad \text { and } \quad m_{n}\left(\mu_{N}\right)=\frac{\alpha}{N}
$$

we have

$$
r_{n}\left(\nu_{N}\right)=\frac{\beta}{N}+O\left(1 / N^{2}\right) \quad \text { and } \quad R_{n}\left(\mu_{N}, \nu_{N}\right)=\frac{\alpha}{N}+O\left(1 / N^{2}\right),
$$

from which it follows that the limiting measures $(\hat{\mu}, \hat{\nu})$ are determined by

$$
\begin{aligned}
r_{n}(\hat{\nu})=\beta & \text { for all } n \geq 1 \\
R_{n}(\hat{\mu}, \hat{\nu})=\alpha & \text { for all } n \geq 1
\end{aligned}
$$

or equivalently, for all $n \geq 1$,

$$
\begin{aligned}
& m_{n}(\hat{\nu})=\sum_{k=1}^{n} t_{k}^{n} \beta^{k} \\
& m_{n}(\hat{\mu})=\sum_{k, l \geq 0} s_{k, l}^{n} \alpha^{k} \beta^{l} .
\end{aligned}
$$

For $\hat{\nu}$, this gives the free Poisson distribution, see [VDN, Maa, Spe1]. The formula for the moments of $\hat{\mu}$ was also derived in [BSp].

As before, we want to calculate the generating power series in the moments

$$
f(z):=\sum_{n=0}^{\infty} m_{n}(\hat{\nu}) z^{n} \quad \text { and } \quad F(z):=\sum_{n=0}^{\infty} m_{n}(\hat{\mu}) z^{n} .
$$


Since $f(z)$ is of eminent importance for the determination of $F(z)$, we will briefly derive its form, although this may also be found in [Maa, VDN]. By part (i) of Lemma 4.2, we obtain

$$
\begin{aligned}
f(z) & =1+\sum_{n=1}^{\infty}\left(\sum_{k=1}^{n} t_{k}^{n} \beta^{k}\right) z^{n} \\
& =1+\sum_{n=1}^{\infty} \sum_{k=1}^{n}\left(t_{k-1}^{n-1}+\sum_{r=2}^{n} \sum_{i=1}^{r-1} t_{i}^{r-1} t_{k-i}^{n-r}\right) \beta^{k} z^{n} \\
& =1+\beta z \sum_{n=1}^{\infty} \sum_{k=1}^{n} t_{k-1}^{n-1} \beta^{k-1} z^{n-1}+h(z) \\
& =1+\beta z f(z)+h(z),
\end{aligned}
$$

where

$$
h(z)=z \sum_{n=1}^{\infty} \sum_{k=1}^{n} \sum_{r=2}^{n} \sum_{i=1}^{r-1} t_{i}^{r-1} \beta^{i} z^{r-1} t_{k-i}^{n-r} \beta^{k-i} z^{n-r}=z(f(z)-1) f(z),
$$

thus

$$
f(z)=1+\beta z f(z)+z(f(z)-1) f(z) .
$$

This can be resolved to give (note $f(0)=1$ )

$$
f(z)=\frac{1-(\beta-1) z-\sqrt{(1-(\beta-1) z)^{2}-4 z}}{2 z}
$$

or

$$
g(z)=\frac{1}{z} f\left(\frac{1}{z}\right)=\frac{z+(1-\beta)-\sqrt{(z-(1+\beta))^{2}-4 \beta}}{2 z} .
$$

For the determination of $F(z)$, we use part (iii) and (ii) of Lemma 4.2. We have

$$
\begin{aligned}
F(z) & =1+\sum_{n=1}^{\infty} \sum_{l, k \geq 0} s_{k+1, l}^{n} \alpha^{k+1} \beta^{l} z^{n} \\
& =1+\sum_{n=1}^{\infty} \sum_{l, k \geq 0} \sum_{r=1}^{n} \sum_{j=0}^{l}\left(s_{1, j}^{r} \alpha \beta^{j} z^{r}\right)\left(s_{k, l-j}^{n-r} \alpha^{k} \beta^{l-j} z^{n-r}\right) \\
& =1+\sum_{j \geq 0} \sum_{r \geq 1}\left(s_{1, j}^{r} \alpha \beta^{j} z^{r}\right) F(z)
\end{aligned}
$$




$$
\begin{aligned}
& =1+F(z)\left\{\sum_{j \geq 0} s_{1, j}^{1} \alpha \beta^{j} z+\frac{\alpha}{\beta} z \sum_{j \geq 0} \sum_{r \geq 2} t_{j+1}^{r-1} \beta^{j+1} z^{r-1}\right\} \\
& =1+F(z)\left\{\alpha z+\frac{\alpha}{\beta} z(f(z)-1)\right\},
\end{aligned}
$$

which implies

$$
F(z)=\frac{\beta}{\beta-\alpha z(f(z)-1+\beta)} .
$$

This yields for the Cauchy-transform $G(z)=1 / z F(1 / z)$ of $\hat{\mu}$ after some calculations the expression

$$
G(z)=\frac{z(2 \beta-\alpha)+\alpha(1-\beta)-\alpha \sqrt{(z-(1+\beta))^{2}-4 \beta}}{2 z[z(\beta-\alpha)+\alpha(1-\beta+\alpha)]} .
$$

The Stieltjes inversion formula [AGl] gives then, after some computations, the distribution as stated in the theorem.

\section{Remarks.}

(1) Again, it is quite instructive to write the Cauchy-transforms as infinite continued fractions, namely

$$
g(z)=\frac{1}{z+(1-\beta)-\frac{z}{z+(1-\beta)-\frac{z}{z+(1-\beta)-\frac{z}{\ldots}}}}
$$

and

$$
G(z)=\frac{1}{z+\frac{\alpha}{\beta}(1-\beta)-\frac{\frac{\alpha}{\beta} z}{z+(1-\beta)-\frac{z}{z+(1-\beta)-\frac{z}{\cdots}}} .}
$$

(2) Note that our formula for $G(z)$ in [BSp] was wrong.

(3) In Fig. 2, we show the Poisson limit distribution $\pi_{\alpha, \beta}$ for $\alpha=1$ and for six different values of $\beta$. 


\section{Analytic description of the c-free convolution.}

In Sect. 3, we described the c-free convolution from a combinatorial point of view by presenting the connection between moments and free and c-free cumulants; the convolution is then characterized by the fact that the cumulants are linear under convolution.

For an analytic description one wants to translate this connection into a functional relation between the corresponding power series, i.e. instead of a collection of moments or cumulants one prefers to deal with one respective analytic function containing the same information. This has the advantage that an analytic machinery is usual more powerful than a mere combinatorial description and it may serve as a starting point for the treatment of measures with unbounded support.

Thus, given a pair $(\mu, \nu) \in \mathcal{M}^{2}$, we define the following power series (formally, we put $r_{0}=R_{0}=0$ )

$$
\begin{aligned}
A(z) & :=\sum_{n=1}^{\infty} r_{n}(\nu) z^{n} \\
B(z) & :=\sum_{n=0}^{\infty} m_{n}(\nu) z^{n}=1+\sum_{n=1}^{\infty} m_{n}(\nu) z^{n} \\
C(z) & :=\sum_{n=1}^{\infty} R_{n}(\mu, \nu) z^{n} \\
D(z) & :=\sum_{n=0}^{\infty} m_{n}(\mu) z^{n}=1+\sum_{n=1}^{\infty} m_{n}(\mu) z^{n} .
\end{aligned}
$$

Since $r_{n}$ and $R_{n}$ are additive under c-free convolution, one has for $(\mu, \nu)=$ $\left(\mu_{1}, \nu_{1}\right) \boxplus\left(\mu_{2}, \nu_{2}\right)$

$$
\begin{aligned}
A_{\nu}(z) & =A_{\nu_{1}}(z)+A_{\nu_{2}}(z) \\
C_{(\mu, \nu)}(z) & =C_{\left(\mu_{1}, \nu_{1}\right)}(z)+C_{\left(\mu_{2}, \nu_{2}\right)}(z)
\end{aligned}
$$

and it remains to derive the connection between $A(z)$ and $C(z)$ on one side and $B(z)$ and $D(z)$ on the other side. Since $\nu=\nu_{1} \boxplus \nu_{2}$ is nothing else than the free convolution, the relation between $A(z)$ and $B(z)$ is given in [Voi2, Spe2].

Theorem 5.1. With the above definitions we have

$$
A[z B(z)]+1=B(z) \quad \text { or } \quad B\left[\frac{z}{1+A(z)}\right]=1+A(z)
$$

and

$$
C[z B(z)] \cdot D(z)=(D(z)-1) \cdot B(z) .
$$


Proof. We only have to show the relation between $B(z), C(z)$, and $D(z)$. The crucial relation is the definition of the c-free cumulants $R_{k}=R_{k}(\mu, \nu)$ by

$$
m_{n}(\mu)=\sum_{k=1}^{n} \sum_{\substack{l(1), \ldots, l(k) \geq 0 \\ l(1)+\cdots+l(k)=n-k}} R_{k} \cdot m_{l(1)}(\nu) \cdot \ldots \cdot m_{l(k-1)}(\nu) \cdot m_{l(k)}(\mu) .
$$

Now define

$$
\hat{C}(z):=\frac{1}{z} C(z)=\sum_{n=1}^{\infty} R_{n} z^{n-1}
$$

Then we have

$$
\begin{aligned}
& \hat{C}[z B(z)] \cdot D(z) \\
& =\sum_{k=1}^{\infty} R_{k}\left(\sum_{l=0}^{\infty} m_{l}(\nu) z^{l}\right)^{k-1}\left(\sum_{l(k)=0}^{\infty} m_{l(k)}(\mu) z^{l(k)}\right) z^{k-1} \\
& =\sum_{k=1}^{\infty} R_{k} \sum_{\substack{l(1), \ldots, l(k) \geq 0 \\
m_{l(1)}}} m^{\infty} \ldots \cdot m_{l(k-1)}(\nu) \cdot m_{l(k)}(\mu) \cdot z^{l(1)+\cdots+l(k)+(k-1)} \\
& =\sum_{n=1}^{\infty} \sum_{k=1}^{n} \sum_{\substack{l(1), \ldots, l(k) \geq 0 \\
l(1)+\cdots+l(k)=n-k}} R_{k} \cdot m_{l(1)}(\nu) \cdot \ldots \cdot m_{l(k-1)}(\nu) \cdot m_{l(k)}(\mu) \cdot z^{n-1} \\
& =\sum_{n=1}^{\infty} m_{n}(\mu) z^{n-1} \\
& =\frac{1}{z}(D(z)-1)
\end{aligned}
$$

hence

$$
\frac{1}{z B(z)} C[z B(z)] \cdot D(z)=\frac{1}{z}(D(z)-1)
$$

which gives the assertion.

Instead of dealing with the generating power series $B(z)$ and $D(z)$ in the moments it is usually more convenient to replace them by the corresponding Cauchy-transforms

$$
g(z)=1 / z \cdot B(1 / z) \text { and } G(z)=1 / z \cdot D(1 / z) .
$$

If we also replace the series $A(z)$ and $C(z)$ by the $r / R$-transforms

$$
r(z)=A(z) / z \quad \text { and } \quad R(z)=\hat{C}(z)=C(z) / z
$$


then our main result can be rewritten as follows.

Theorem 5.2. With the notations as above we have

$$
g(z)=\frac{1}{z-r[g(z)]} \quad \text { or } \quad g\left[r(z)+z^{-1}\right]=z
$$

and

$$
G(z)=\frac{1}{z-R[g(z)]}
$$

Examples.

1) Gaussian distribution as in Theorem 4.3.

We have

$$
r(z)=\beta^{2} z \quad \text { and } \quad R(z)=\alpha^{2} z
$$

which gives

$$
g(z)=\frac{1}{z-\beta^{2} g(z)} \quad \text { and } \quad G(z)=\frac{1}{z-\alpha^{2} g(z)}
$$

which agrees with our calculations in Sect. 4. Note that in our proof of Theorem 4.3 we used other combinatorial identities than here. Our current machinery does not reproduce the proof of 4.3 , but it specializes to the formulas given in the remark after 4.3.

2) Poisson distribution as in Theorem 4.4.

We have

$$
r(z)=\beta \frac{1}{1-z} \quad \text { and } \quad R(z)=\alpha \frac{1}{1-z}
$$

which gives

$$
g(z)=\frac{1}{z-\beta \frac{1}{1-g(z)}} \quad \text { or } \quad g(z)=\frac{1}{z+(1-\beta)-z g(z)}
$$

and

$$
G(z)=\frac{1}{z-\alpha \frac{1}{1-g(z)}} \quad \text { or } \quad G(z)=\frac{1}{z+\frac{\alpha}{\beta}(1-\beta)-\frac{\alpha}{\beta} z g(z)}
$$


in agreement with our calculations in Sect. 4.

Remark. In the case of the boolean convolution $\left(\mu, \delta_{0}\right)=\left(\mu_{1}, \delta_{0}\right) \boxplus\left(\mu_{2}, \delta_{0}\right)$, which we mentioned in Remark 2 in Sect. 3 , we have $g(z)=g_{\delta_{0}}(z)=1 / z$ and our formula in Theorem 5.2 reduces to

$$
G(z)=\frac{1}{z-K(z)} \quad \text { with } \quad K(z)=R(1 / z)
$$

This simple formula reflects the simple structure of the underlying lattice of interval partitions and offers the possibility for a far reaching analytic treatment of the boolean convolution, in this respect see [Wor, SpW]. 

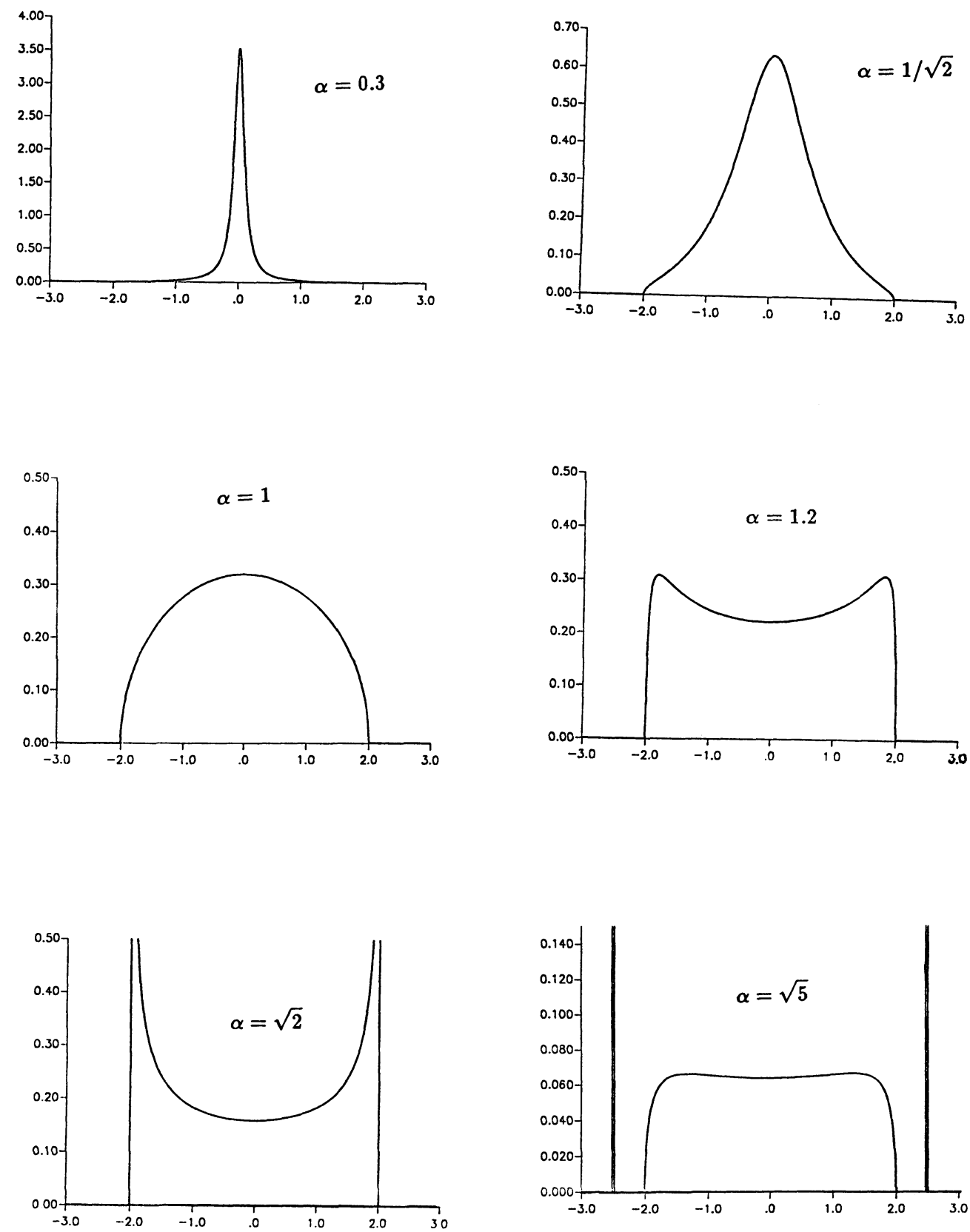

Figure 1. c-free Gaussian distribution $\nu_{\alpha, \beta}$ for fixed $\beta=1$ and six different values of $\alpha$; vertical double lines indicate $\delta$-peaks. 

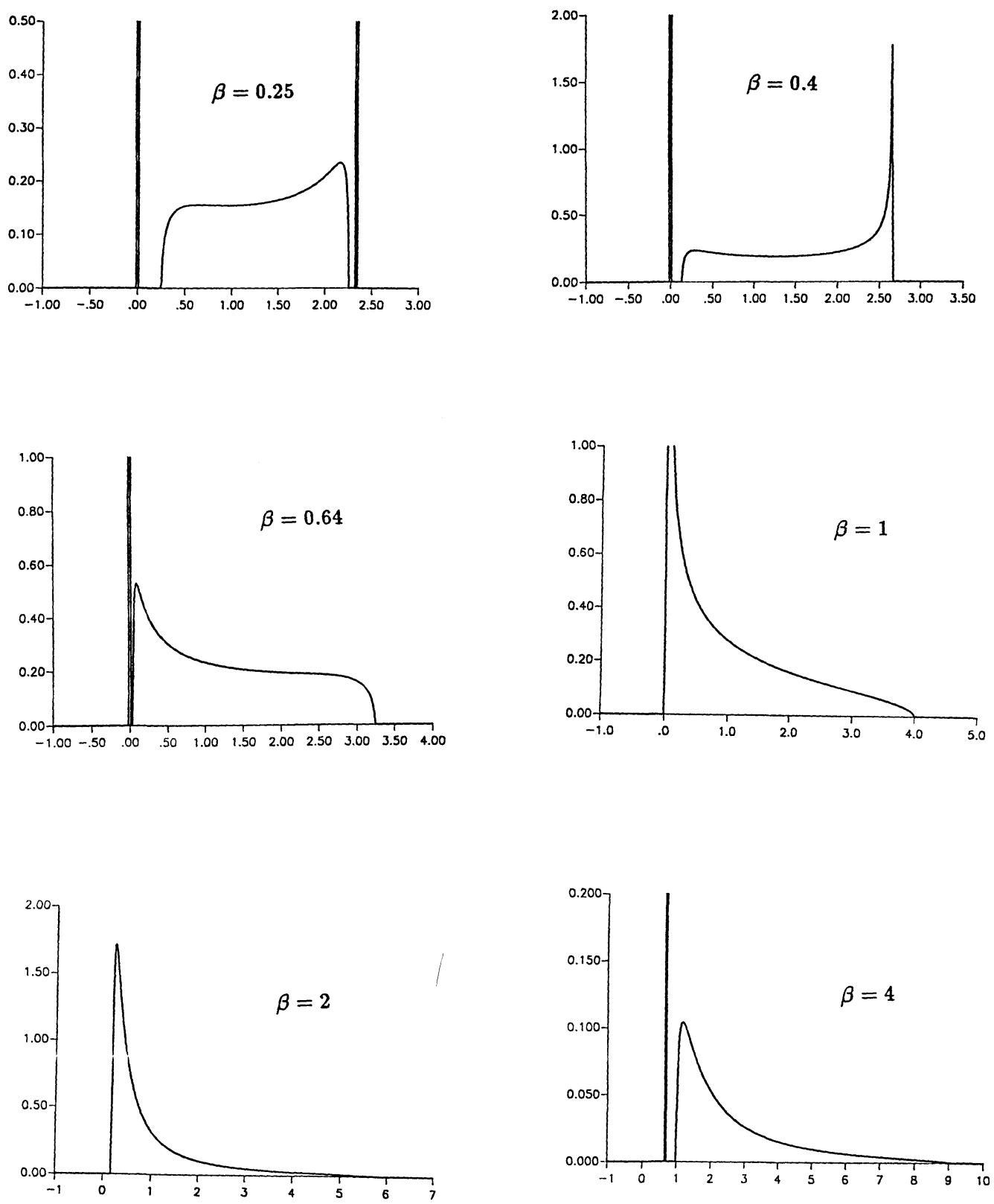

Figure 2. c-free Poisson distribution $\pi_{\alpha, \beta}$ for fixed $\alpha=1$ and six different values of $\beta$; vertical double lines indicate $\delta$-peaks; note that the $\delta$-peak at $z_{0}$ lies first on the right side of the continuous spectrum, then it dissapears and reappears again on the left side of the continuous spectrum. 


\section{References}

[AGl] N.I. Achieser and I.M. Glasmann, Theorie der linearen Operatoren im Hilbert Raum, Akademie-Verlag, Berlin, 1960.

[Bia] P. Biane, Some properties of crossings and partitions, 1994, preprint.

[Boz1] M. Bożejko, Positive definite functions on the free group and the non commutative Riesz product, Boll. Un. Mat. Ital., 5 A (1986), 13-21.

[Boz2] Uniformly bounded representations of free groups, J. Reine Angew. Math., 377 (1987), 170-186.

[BSp] M. Bożejko and R. Speicher, $\psi$-independent and symmetrized white noises Quantum Probability and Related Topics, (L. Accardi, ed.), World Scientific, Singapore, VI (1991), 219-236.

[CDu] T. Cabanal-Duvillard, Variation quantique sur l'independance: La $\sigma$-independance, 1993, preprint.

[Ede1] P.H. Edelman, Chain Enumeration and Non-Crossing Partitions, Discr. Math., 31 (1980), 171-180.

[Ede2] — Multichains, Non-Crossing Partitions and Trees, Discr. Math., 40 (1982), 171-179.

[Gir] W.L. Girko, Random Matrices, Kiev, 1975, (Russian).

[Haa] U. Haagerup, An Example of a Non Nuclear $C^{*}$-Algebra which has the Metric Approximation Property, Invent. Math., 50 (1979), 279-293.

[HiP] P. Hilton and J. Pederson, Catalan Numbers, Their Generalization, and Their Uses, Math. Intelligencer, 13(2) (1991), 64-75.

[Kre] G. Kreweras, Sur les partitions non croisees d'un cycle, Discr. Math., 1 (1972), 333-350.

[Maa] H. Maassen, Addition of freely independent random variables, J. Funct. Anal., 106 (1992), 409-438.

[Nic] A. Nica, Crossings and embracings of set-partitions and q-analogues of the logarithm of the Fourier transform, Discr. Math., to appear.

[NSp1] P. Neu and R. Speicher, A self-consistent master equation and a new kind of cumulants, Z. Phys. B, 92 (1993), 399-407.

[NSp2] - Non-linear master equation and non-crossing cumulants, Quantum Probability and Related Topics, (L. Accardi, ed.), World Scientific, Singapore, IX (1994), 311-326.

[Pou] Y. Poupard, Etude et denombrement paralleles des partitions non croisees d'un cycle et coupage d'un polygone convexe, Discr. Math., 2 (1972), 279-288.

[Sim] R. Simion, Combinatorial statistics on non-crossing partitions, 1991, preprint.

[SiU] R. Simion and D. Ullman, On the structure of the lattice of non-crossing partitions, Discr. Math., 98 (1991), 193-206.

[Spe1] R. Speicher, A New Example of "Independence" and "White Noise", Probab. Th. Rel. Fields, 84 (1990), 141-159.

[Spe2] - Multiplicative functions on the lattice of non-crossing partitions and free convolution, Math. Ann., 298 (1994), 611-628.

[SpW] R. Speicher and R. Woroudi, Boolean convolution, The Fields Institute Communications, to appear. 
[Voi1] D. Voiculescu, Symmetries of some reduced free product $C^{*}$-algebras, Operator Algebras and their Connection with Topology and Ergodic Theory, Lecture Notes in Mathematics, Springer, Heidelberg, 1132 (1985), 556-588.

[Voi2] , Addition of certain non-commuting random variables, J. Funct. Anal., 66 (1986), 323-346.

[VDN] D. Voiculescu, K. Dykema and A. Nica, Free Random Variables, AMS, 1992.

[vWa] W. von Waldenfels, An approach to the theory of pressure broadening of spectral lines, Lecture Notes in Mathematics, Springer, Heidelberg, 296 (1973), 19-69.

[Wor] R. Woroudi, Boolesche Faltung, Heidelberg, diploma thesis, 1993.

Received October 21, 1994. This work has been supported by Polish National Grant, KBN 4019 (M.B.) and a fellowship from the Deutsche Forschungsgemeinschaft (R.S.).

UNIWERSYTET WROCLAWSKI

Plac GrunWaldzKi $2 / 4$

50-384 Wroclaw, Poland

E-mail address: bozejko@math.uni.wroc.pl

AND

UNIVERSITÄT HEIDELBERG

IM NEUENHEIMER FELD 294

D-69120 HeIDelberg, Germany

E-mail address: michael.leinert@urz.uni-heidelberg.de roland.speicher@urz.uni-heidelberg.de 
Mogens L. Hansen and Richard V. Kadison, Banach algebras with uni-

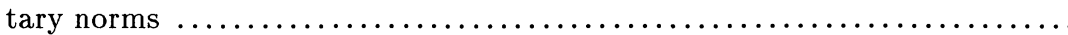

Xin-hou Hua, Sharing values and a problem due to C.C. Yang ..........

Jing-Song Huang, Harmonic analysis on compact polar homogeneous

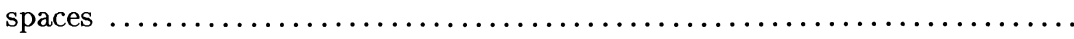

Min-Jei Huang, Commutators and invariant domains for Schrödinger prop-

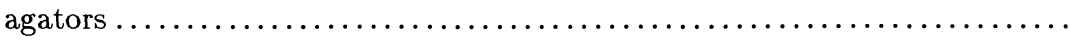

Hisao Kato, Chaos of continuum-wise expansive homeomorphisms and dy-

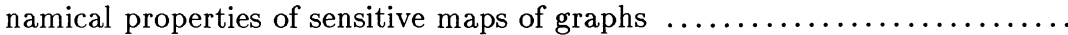

Oliver Küchle, Some properties of Fano manifolds that are zeros of sections in homogeneous vector bundles over Grassmannians ...................

Xin Li and Francisco Marcellan, On polynomials orthogonal with respect to Sobolev inner product on the unit circle $\ldots \ldots \ldots \ldots \ldots \ldots \ldots \ldots \ldots \ldots$. . . . . . .

Steven Liedahl, Maximal subfields of $Q(i)$-division rings .............. 147

Alan L.T. Paterson, Virtual diagonals and $n$-amenability for Banach algebras ....................................................

Claude Schochet, Rational Pontryagin classes, local representations, and

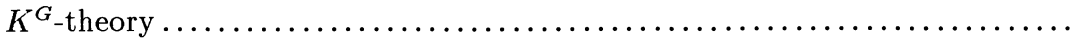

Sandra L. Shields, An equivalence relation for codimension one foliations of 3-manifolds

D. Siegel and E. O. Talvila, Uniqueness for the $n$-dimensional half space

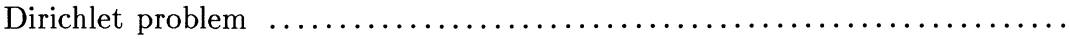

Aleksander Simonič, A Construction of Lomonosov functions and applica-

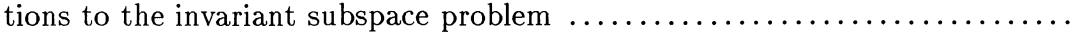

Endre Szabó, Complete intersection subvarieties of general hypersurfaces.. 


\section{PACIFIC JOURNAL OF MATHEMATICS}

Volume $175 \quad$ No. $2 \quad$ October 1996

Mean-value characterization of pluriharmonic and separately harmonic functions

LeV Abramovich AĬZEnberg, CARlos A. Berenstein and L.

WERTHEIM

Convergence for Yamabe metrics of positive scalar curvature with integral bounds on 307 curvature

\section{KAZUO AKUTAGAWA}

Generalized modular symbols and relative Lie algebra cohomology

\section{AVNER DOLNICK ASH and DAVID GINZBURG}

Convolution and limit theorems for conditionally free random variables

MaReK BożejKo, Michael LEINERT and Roland SPEICHER

$L^{p}$-bounds for hypersingular integral operators along curves

SHARAD CHANDARANA

On spectra of simple random walks on one-relator groups. With an appendix by Paul Jolissain

Pierre-Alain Cherix, Alain J. Valette and Paul Jolissaint

Every stationary polyhedral set in $\mathbf{R}^{n}$ is area minimizing under diffeomorphisms

JAIGYOUNG CHOE

Ramanujan's master theorem for symmetric cones

Hongming Ding, Kenneth I. GRoss and Donald Richards

On norms of trigonometric polynomials on SU(2)

AnTHONy H. DOOLEY and SANJiV Kumar GuPTA

On the symmetric square. Unit elements

YUVAL ZVI FLICKER

Stable constant mean curvature surfaces minimize area

KARSTEN GROSSE-BRAUCKMANN

Banach algebras with unitary norms

Mogens LEMVIG HANSEN and RiCHARD VinCENT KADISON

Harmonic analysis on compact polar homogeneous spaces

JING-SONG HUANG

Uniqueness for the $n$-dimensional half space Dirichlet problem

DAVID SIEGEL and ERIK O. TALVILA 